\title{
Dynamic Molecular Switches Drive Negative Memristance Emulating Synaptic Behaviour
}

\section{Christian Nijhuis ( $\nabla$ c.a.nijhuis@utwente.nl)}

University of Twente https://orcid.org/0000-0003-3435-4600

\section{Yulong Wang}

Department of Chemistry, National University of Singapore, 3 Science Drive 3, Singapore 117543. https://orcid.org/0000-0001-9441-0497

\section{Qian Zhang}

national university of singapore

\section{Hippolyte Astier}

Department of Chemistry, National University of Singapore

\section{Cameron Nickle}

University of Central Florida

\section{Ziyu Zhang}

Department of Chemistry, National University of Singapore

\section{Andrea Leoncini}

national university of singapore

Dongchen Qi

Queensland University of Technology https://orcid.org/0000-0001-8466-0257

\section{Yingmei Han}

Department of Chemistry, National University of Singapore

\section{Enrique del Barco}

University of Central Florida https://orcid.org/0000-0002-5763-0076

\section{Damien Thompson}

University of Limerick https://orcid.org/0000-0003-2340-5441

\section{Article}

Keywords:

Posted Date: January 20th, 2022

DOI: https://doi.org/10.21203/rs.3.rs-1156230/v1

License: (9) (1) This work is licensed under a Creative Commons Attribution 4.0 International License. 
Additional Declarations: There is NO Competing Interest.

Version of Record: A version of this preprint was published at Nature Materials on November 21st, 2022. See the published version at https://doi.org/10.1038/s41563-022-01402-2. 


\section{Dynamic Molecular Switches Drive Negative}

\section{Memristance Emulating Synaptic Behaviour}

Yulong Wang, ${ }^{1 \dagger}$ Qian Zhang, ${ }^{1 \dagger}$ Hippolyte P.A.G. Astier ${ }^{1 \dagger}$, Cameron Nickle, ${ }^{2 \dagger}$ Ziyu Zhang, ${ }^{1}$ Andrea Leoncini, ${ }^{1}$ Dong-Cheng Qi, ${ }^{3}$ Yingmei Han, ${ }^{1}$ Enrique del Barco ${ }^{2 *}$, Damien Thompson ${ }^{4 *}$ and Christian A. Nijhuis ${ }^{1,5,6^{*}}$

${ }^{1}$ Department of Chemistry, National University of Singapore, 3 Science Drive 3, Singapore 117543, Singapore.

${ }^{2}$ Department of Physics, University of Central Florida, Orlando, Florida 32816 - USA. ${ }^{3}$ School of Chemistry, Physics and Mechanical Engineering, Queensland University of Technology, Brisbane, Queensland 4001, Australia.

${ }^{4}$ Department of Physics, Bernal Institute, University of Limerick, Limerick V94 T9PX, Ireland.

${ }^{5}$ Centre for Advanced 2D Materials and Graphene Research Center, National University of Singapore, 6 Science Drive 2, Singapore 117546, Singapore.

${ }^{6}$ Hybrid Materials for Opto-Electronics Group, Department of Molecules and Materials, MESA+ Institute for Nanotechnology and Center for Brain-Inspired Nano Systems, Faculty of Science and Technology, University of Twente, 7500 AE Enschede, The Netherlands.

${ }^{\dagger}$ These authors contributed equally to this work

*Authors to whom correspondence should be addressed: c.a.nijhuis@utwente.nl, damien.thompson@ul.ie and delbarco@ucf.edu. 
Abstract: To realise molecular scale electrical operations beyond the von Neumann bottleneck, new types of multi-functional switches are needed that mimic selflearning or neuromorphic computing by dynamically toggling between multiple operations that depend on their past. Here we report a molecule that switches from high to low conductance states with massive negative memristive behaviour that depends on the drive speed and number of past switching events, with all measurements fully modelled using atomistic and analytical models. This dynamic molecular switch (DMS) emulates synaptic behaviour and Pavlovian learning, all within a $2.4 \mathrm{~nm}$ thick layer that is three orders of magnitude thinner than a neuronal synapse. The DMS provides all fundamental logic gates necessary for deep learning because of its time-domain and voltage-dependent plasticity. The synapse-mimicking multi-functional DMS represents adaptable molecular scale hardware operable in solid-state devices opening a pathway to simplify dynamic complex electrical operations encoded within a single ultra-compact component. 


\section{Introduction}

Inspired by the energy efficiency of brains and the ever-increasing demand for miniaturised electronics, there is a drive to develop devices that mimic the dynamic character of neurons and synapses to create trainable, adaptive computing networks or new hardware for deep learning for various applications, including pattern recognition, classification, or to realise non-von Neumann neuromorphic computation ${ }^{1-5}$. Neuromorphic computing revolves around emulating synaptic plasticity in electronic systems. Such operations are currently performed using complex, energy-inefficient silicon-based circuits with large footprints, or mesoscale memristive systems based on ferroelectric $^{6}$ or phase change materials ${ }^{7}$, filaments, or migration of dopants ${ }^{1,3,8}$. In this context, molecular switches are appealing because of their inherently small size but molecular switches so far are static, meaning they switch between fixed on and off states (e.g., magnetic, redox, or conformational states $)^{9-11}$. Here we report a dynamic molecular switch in a tunnel junction that remembers its history, whereby the switching probability and the values of the on/off states continually change. This dynamic switch successfully emulates synaptic behaviour, Pavlovian learning, and, by exploiting the time-domain plasticity of the junctions, mimics all two-terminal logic gates necessary for deep learning. These functionalities are provided within a single molecular layer $(2.4 \mathrm{~nm}$ thick $)$, smaller than a neuron synapse $(\sim 1-10 \mu \mathrm{m})$ by at least three orders of magnitude, and thinner even than the synaptic gap $(\sim 20-40 \mathrm{~nm})$ by an order of magnitude. This dynamic nature represents a new class of multi-functional molecular switch simplifying brain-inspired electronic devices, opening the door to neuromorphic computing at the molecular scale.

One of the goals of neuromorphic electronics is to produce computing systems where the training is done at the hardware level but molecular hardware for doing so 
is not available. Unlike molecular switches studied so far, biological switches, e.g., synapses, are inherently dynamic operating far away from thermodynamic equilibrium $^{12,13}$ and therefore can be trained. In synapses (Fig.1a), information travels in the form of an action potential from a presynaptic neuron to a postsynaptic neuron through the synaptic gap, as regulated by the combined excitatory and inhibitory neural inputs ${ }^{14}$. By coupling different processes each characterised with different time constants, e.g., fast depolarisation coupled to slow diffusion of $\mathrm{Ca}^{2+}$ and neurotransmitters, the synapses can be strengthened or weakened depending on their activity ${ }^{15}$ resulting in synaptic plasticity enabling pulse pair facilitation and depression with spike rate and timing dependent plasticity. To emulate the dynamical behaviour of synapses at the molecular level, we combined fast electron transfer (akin to action potentials and fast depolarisation processes) with slow proton coupling limited by diffusion (akin to the role of $\mathrm{Ca}^{2+}$ or neurotransmitters).

\section{Solid-state dynamic molecular switches}

Fig.1b-d shows the dynamic molecular switch (DMS) we introduce here, incorporated in junctions consisting of a self-assembled monolayer (SAM) supported by a gold bottom electrode in contact with an EGaIn top electrode. The SAM molecule has a 5,6,11,12,17,18-hexaazatrinaphthylene (HATNA) terminus that readily undergoes six successive proton coupled electron transfer (PC-ET) steps for dynamic covalent $\mathrm{N}-\mathrm{H}$ bond formation ${ }^{16,17}$. In the following we indicate the various oxidation states as Hn-HATNA with $n=0-6$. When the molecules are oxidised $(n=0-2)$ and turn on, electron transfer (ET) across the junctions is fast (fast channel, indicated by a blue arrow) resulting in a large increase of the currents flowing across the junction. In parallel to this fast ET process, proton addition steps occur, and N-H 
formation slows down the ET rate (slow channel, dashed red arrow) and turns the junction off when HATNA is progressively reduced $(n=3-6)$. In our experiments, water from the ambient (air, room temperature) is the proton source and so proton transfer is diffusion limited and much slower than ET. This switching from the fast to slow channel occurs at negative bias when the molecules are reduced resulting in a decrease of current and negative differential resistance (NDR). The junctions are oxidised back to their original states by applying a positive bias. Since the ET and proton coupling (PC) steps occur at very different time scales, our junctions can emulate the plastic behaviour of synapses, Pavlovian learning, and all logic gates, simply by changing the applied voltage and the duration of voltage pulses. 


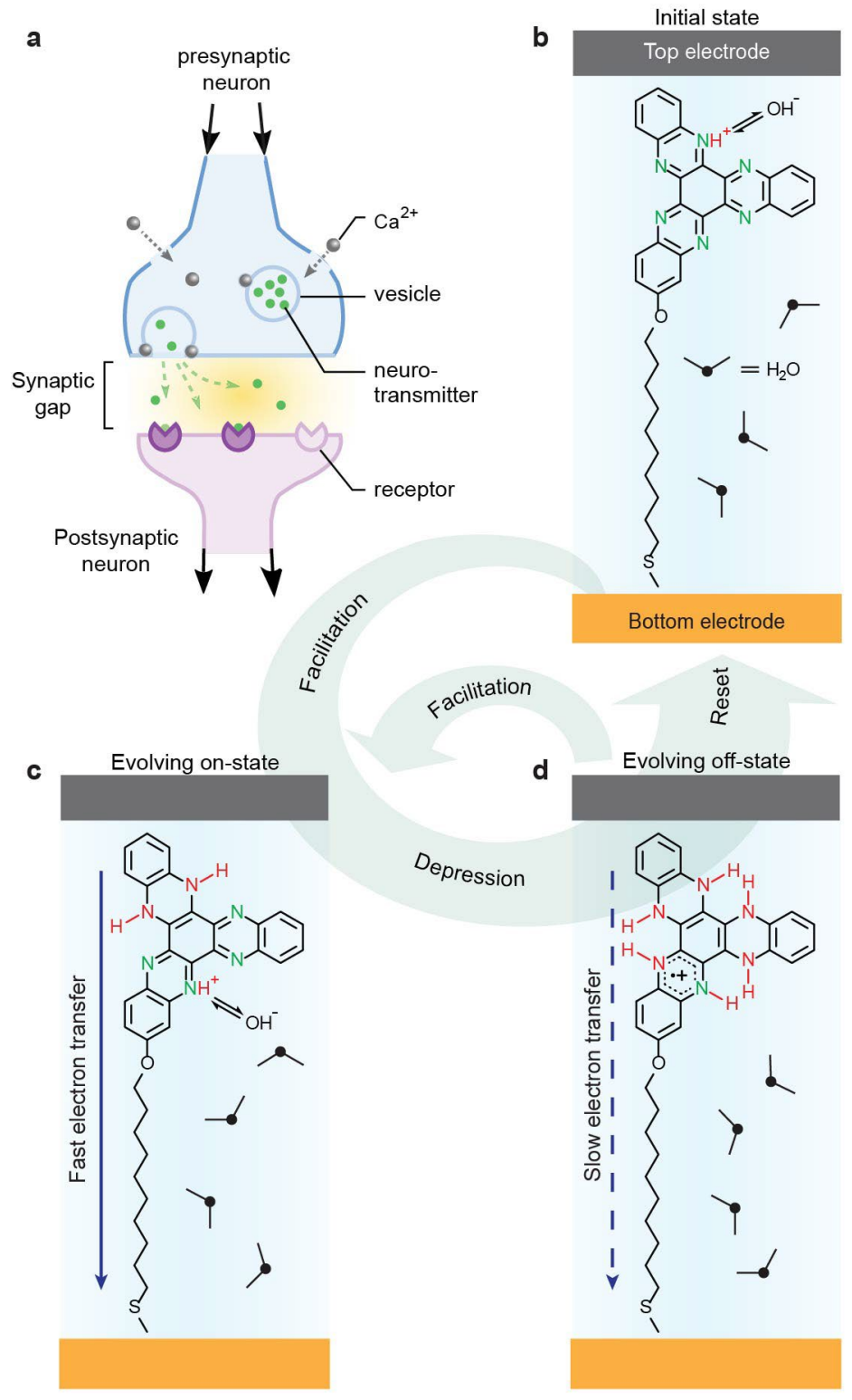

Fig.1. The dynamic molecular junction. a, Schematic of a synapse and the junctions (b-d). The junction consists of a Au bottom electrode and EGaIn top electrode (EGaIn $=$ eutectic alloy of gallium and indium). Solid and dashed blue arrows indicate electron transfer (ET) via coherent tunnelling through the fast and slow channels. The two ET channels are bridged by slow proton coupled electron transfer (PC-ET) where the protons are provided by water molecules in air. 


\section{Proton-coupled charge transport and dynamic covalent bond formation}

We characterised the structure of the SAMs using various techniques (Methods and Supplementary Sections S2 and S3) and conclude that the SAMs are densely packed with all HATNA units confined to the top of the SAM as shown, for instance, by the molecular dynamics simulations (Fig.2a-c). To prove that the SAMs undergo sequential PC-ET via stable intermediates for reliable switching, we recorded a cyclic voltammogram (CV) from a S-C10-HATNA SAM (Fig.2d). Four distinct redox waves are visible which is similar to the results by Ohsaka and co-workers ${ }^{17}$. They proposed that protonation initiates the first PC-ET step followed by ring-by-ring reduction of HATNA (Fig.2e) explaining why the PC-ET cascade appears as distinct redox waves. Our density functional theory (DFT) calculations confirm this mechanism (Supplementary Section S4).

Although the fully reduced SAM has essentially the same supramolecular structure as the SAM in its initial state (Fig.2c), our DFT calculations reveal that the electronic structure of the SAM strongly depends on the oxidation and protonation state of HATNA. Fig.2f shows projected density of states (PDOS) of the molecule during PC-ET following the steps in Fig.2g calculated with DFT. Fig.2g shows that the molecule in the initial state ( $\mathrm{H}_{0}$-HATNA) has large HOMO-LUMO gap $(\mathrm{HOMO}=$ highest occupied molecular orbital, $\mathrm{LUMO}=$ lowest unoccupied molecular orbital) of $2.1 \mathrm{eV}$, but upon initial protonation resulting in (HATNA) $\mathrm{H}^{+}$, a new midgap state appears around $1.1 \mathrm{eV}$ above the Fermi-energy $\left(E_{\mathrm{F}}\right)$ of the $\mathrm{Au}$ electrode providing a low-energy tunnelling channel which defines the on-state of the junction (Figs. $1 \mathrm{~b}$ and $21-\mathrm{m}$ ). This state persists during the first two PC-ET steps resulting in $\mathrm{H}_{\mathrm{n}}$-HATNA with $\mathrm{n}=1,2$ (Fig.1c) but then shifts towards lower energy before disappearing for $\mathrm{H}_{5}$-HATNA and the large conductance gap is restored (Figs. 1d and 
2o-p), turning the junction off (resulting in NDR as explained below). The changes in HOMO, LUMO, and mid-gap energy levels during all proton and electron addition steps are given in Fig.2g, Extended Data Fig. 1, and Supplementary Section S4. 

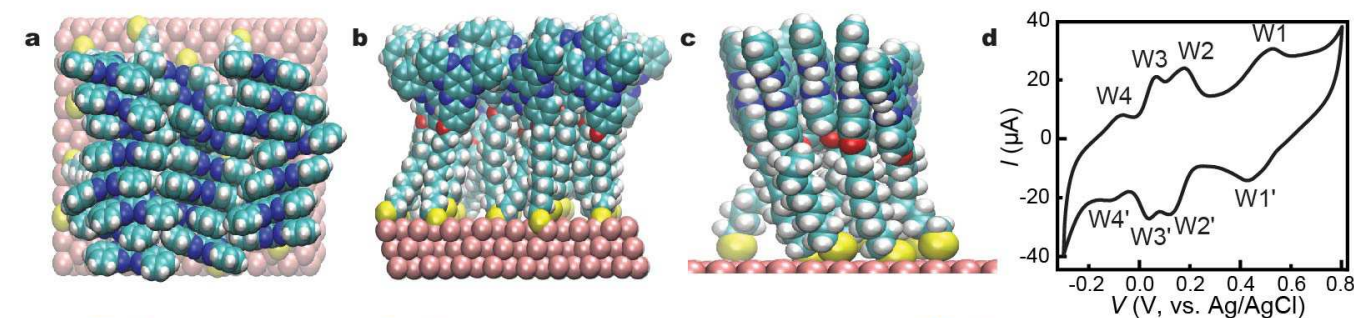

Off state $\square \quad \square$ On state $\square$

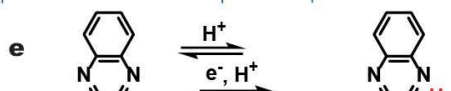
(1)

Ho-HATNA

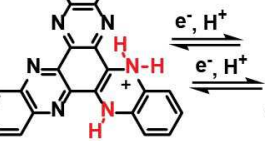

$\left(\mathrm{H}_{2}\right.$-HATNA $) \mathrm{H}^{+}$

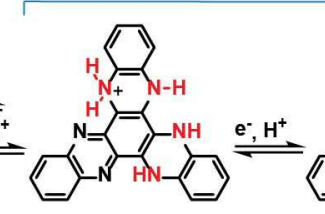

Off states

$V(\mathrm{~V}, \mathrm{vs} . \mathrm{Ag} / \mathrm{AgCl})$
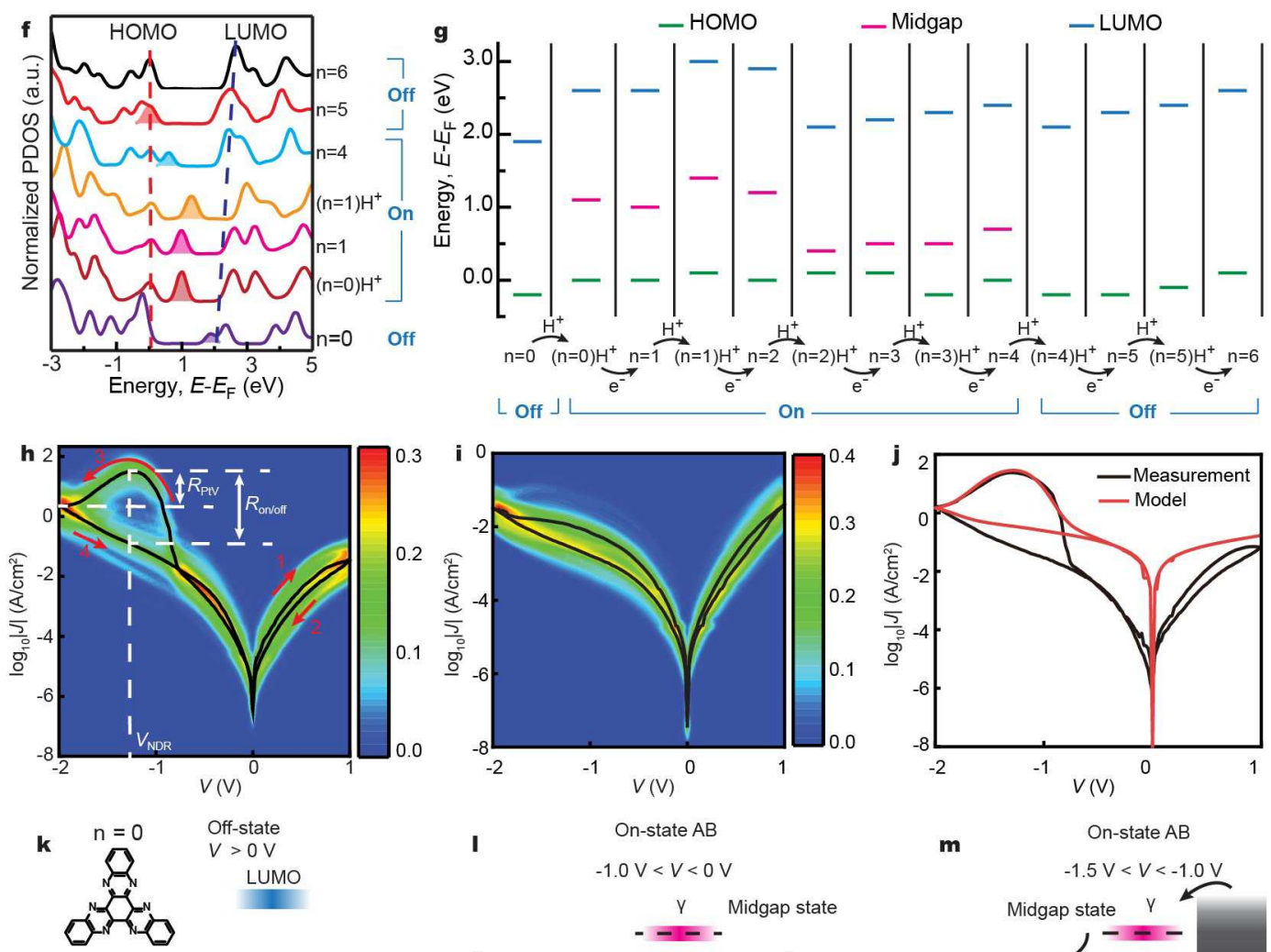

$$
\text { I }
$$

On-state $A B$

I $-1.0 V<V<0 V$

$=$

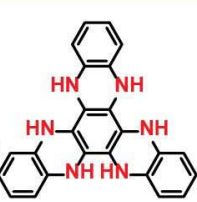

$\int_{\mathrm{NH}}^{\stackrel{\mathrm{e}^{-}, \mathrm{H}^{+}}{\rightleftharpoons}}$

(n)

$\left(\mathrm{H}_{5}\right.$-HATNA) $\mathrm{H}^{+}$

H6-HATNA

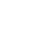


Fig.2. Mechanism of Proton-Coupled Electron Transport. Representative computed structures of $\pi-\pi$ driven supramolecular packing in S-C ${ }_{10}$-HATNA SAMs on $\mathrm{Au}(111)$, from 0.1 microseconds of equilibrated room temperature molecular dynamics with $\mathrm{H}_{0}$-HATNA shown from above (a) and from the side (b), and a zoomin on $\mathrm{H}_{6}$-HATNA shown from the side (c). Carbon, nitrogen, oxygen, sulphur, and hydrogen atoms are coloured cyan, navy, red, yellow, and white. (d) Cyclic voltammogram of a HATNA SAM on Au in contact with $2.0 \mathrm{M}$ aqueous $\mathrm{HClO}_{4}$ recorded at scan rate of $0.5 \mathrm{~V} / \mathrm{s}$ showing four redox-waves labelled W1-4. e Proposed proton-coupled electron transport (PC-ET) mechanism. f, Calculated projected density of states (PDOS) on the molecule of HATNA SAMs on Au(111) for selected oxidation and protonation states (see Extended Data Fig. 1 for all states) and (g) the corresponding HOMO, LUMO and mid-gap energy levels for all states. The oxidation state is indicated by $\mathrm{n}$ and the protonation states are indicated by $\mathrm{H}^{+}$(Supplementary Fig. S6). h, Heatmap of $\log _{10}|J| v s . V\left(24^{\circ} \mathrm{C}\right.$, relative humidity $=60 \%$ in ambient conditions), and (i) Heatmap of $\log _{10}|J| v s . V$ measured under an atmosphere of compressed air with $10 \mathrm{ppm}$ water. The black line is log-median average curve $\left.\left(<\log _{10}|J|\right\rangle_{\mathrm{m}} v s . V\right)$. $\mathbf{j}$, Representative dataset with a fit to the theoretical model (fitting parameters given in Supplementary Table S10). k-p, Model of the dynamic switch. Energy level diagrams of the off $(\mathbf{k}, \mathbf{n}-\mathbf{p})$ and on states $(\mathbf{l}, \mathbf{m})$ of the junctions where tunnelling is mediated by the acceptor level lying above $E_{\mathrm{F}}$ (blue bar), which is the LUMO at low negative or positive bias (k-l) when off-resonant tunnelling dominates but falls in the conduction window at elevated negative bias when the low-lying midgap state forms because of PC-ET $(\mathbf{m})$. The rate equations describe coherent ET tunnelling (horizontal transitions) coupled to slow PC steps (vertical transitions). H6HATNA can be reset into Ho-HATNA by applying a positive $V(\mathbf{n})$. PC increases the tunnelling barrier height of the ET channel by an interaction energy $\chi$ which decreases the molecule-electrode coupling strength $\gamma$ by a factor $\kappa$ (resulting in a broad LUMO) (o) resulting in NDR. The LUMO in the off state is energetically accessible only at large negative bias (p). Note: Although the DFT calculations in panels $f$ and g suggest that the HOMO level is close in energy to $E_{\mathrm{F}}$, the UPS data indicate that the HOMO level is $2.1 \mathrm{eV}$ below the Fermi level of the Au electrode (Supplementary Section S3.2). Given the common problem of level alignment in DFT calculations ${ }^{18}$, we rely on the UPS data to properly position the levels with respect to the electrode work functions in our energy level diagrams shown in panels k-p.

\section{Negative differential memristance}

Fig.2h shows the $J(\mathrm{~V})$ current density-voltage response from the $\mathrm{Au}-\mathrm{S}-\mathrm{C}_{10}-$ HATNA//Ga2 $\mathrm{O}_{3} / \mathrm{EGaIn}$ junctions (see Methods for details). The large hysteric NDR peak can be explained by following the applied voltage in steps $1 \rightarrow 4$ as indicated. Step1: the voltage increases to $+1.0 \mathrm{~V}$ which ensures the HATNA moieties are oxidised $(n=0)$. Step2: voltage is reduced and around $-0.9 \mathrm{~V}$ the current sharply increases as an empty molecular state enters the bias window and the mechanism of 
charge transport changes from off (Fig.2k) to on resonant tunnelling (Fig.2l-m). The PDOS data indicate that the lowest available tunnelling level is the mid-gap state associated with (HATNA) $\mathrm{H}^{+}$, which is likely considering the basic nature of HATNA. Step3: with increasing negative bias, the junctions progressively turn on forming $\mathrm{H}_{\mathrm{n}}$ HATNA with $n=1,2$ (which have this mid-gap state), but turn off at large negative bias when molecules are reduced up to $\mathrm{H}_{n}$-HATNA with $n=5,6$ (which lack the midgap state) resulting in reduction of current (Fig.2n-p) and consequential NDR. Step4: During retrace from -2 to $0 \mathrm{~V}$, junctions remain in the off state resulting in large hysteretic NDR with a memory (i.e., negative memristance). Only at positive bias, the $\mathrm{H}_{\mathrm{n}}$-HATNA moieties are oxidised to HATNA $(\mathrm{n}=0)$ when the HOMO falls inside the conduction window and the cycle restarts (Fig.2n). The value of $R \mathrm{PtV}$ is $13.7 \pm 3.5$ (at $10 \mathrm{mV} / \mathrm{s}$ ) and the value of $R_{\text {on } / \mathrm{off}}$ is $2.64 \pm 1.41 \times 10^{2}$ which are the among the highest values recorded in molecular junctions ${ }^{19}$.

\section{Theoretical model for NDR and dynamic switches}

There are currently no $a b$ initio theories available that can model the dynamical behaviour of junctions, but the analytical model developed by Migliore and Nitzan ${ }^{20}$ captures all our observations as illustrated in Fig. $2 \mathrm{k}-\mathrm{p}$ in the form of energy level diagrams which were constructed based on our DFT (Fig.2f-g) and spectroscopy

results (Supplementary Section S3.2). The model helps us identify the switching regimes in detail (as demonstrated previously for other switches ${ }^{21}$ ), and more importantly, establishes the mechanism of the time and applied-voltage dependency of the dynamical behaviour of the switching. The model describes simultaneous fast ET and slow PC processes in a molecular junction that lead to switching between fast and slow ET channels (Fig.1) as described in detail in Supplementary Section S6. No 
molecular orbitals are available for charge transport at low bias (Fig.21) but at intermediate negative bias (step 3, Fig.2h and Fig.2m) the mid-gap state enters the conduction window, increasing the ET rates of the on state $\left(R_{A B}\right.$ and $R_{B A}$ defined in Fig.2l-m) after addition of one $\mathrm{H}^{+}$, i.e., $\mathrm{H}_{0}-\mathrm{HATNA} \rightarrow\left(\mathrm{H}_{0}-\mathrm{HATNA}\right) \mathrm{H}^{+}$. This protonation step changes the tunnelling barrier height defined by the LUMO to that defined by the mid-gap state as indicated by $\chi$ (Fig.2o-p) and the molecule-electrode coupling strength (given by $\gamma$ and factor $\kappa$ ). Thus, $\chi$ defines the mid-gap to LUMO energy difference which is $2.1 \mathrm{eV}$ (Supplementary Section S6) in good agreement with DFT (1.5-2.0 eV, Fig.2g). At high bias, the HATNA moieties are progressively reduced and junctions turn off again which lowers the ET rates $R_{\overline{A B}}$ and $R_{\overline{B A}}$ (defined in Fig.2o-p) resulting in NDR. In parallel, PC $\left(R_{+P C}\right.$ and $\left.R_{-P C}\right)$ occurs at much slower rates than ET across both the fast and slow channels (i.e., $R_{+P C} \ll R_{A B}, R_{\overline{A B}}$ ), but PC is responsible for switching. Therefore, the ET rates $\left(R_{A B}, R_{B A}\right.$ and $\left.R_{\overline{A B}}, R_{\overline{B A}}\right)$ and associated currents in the on $\left(I^{A B}\right)$ and off $\left(I^{\overline{A B}}\right)$ state are modelled with a single-level Landauer model ${ }^{22}$ while the much slower PC steps $\left(R_{-P C}, R_{+P C}\right)$ are described by Marcus theory ${ }^{23}$ routinely used to model PC-ET steps ${ }^{24-26}$. Following Migliore and Nitzan's model ${ }^{20}$, the current that flows across the junctions is calculated from the probability of the junctions to be in the on $\left(P^{A B}\right)$ or off $\left(P^{\overline{A B}}\right)$ state as given by

$$
I(V)=P^{A B}(V) I^{A B}(V)+P^{\overline{A B}}(V) I^{\overline{A B}}(V) \quad(E q n 1)
$$

where $P^{A B}(V)=1-P^{\overline{A B}}(V)$. The overall dynamical probability of the junction is governed by the slow protonation rates $\left\langle R_{+P C}>\right.$ and $\left\langle R_{-P C}\right\rangle$

$$
\frac{\mathrm{d} P^{A B}}{\mathrm{~d} t}=\left(1-P^{A B}\right)<R_{+P C}>-P^{A B}<R_{-P C}>\quad(E q n 2)
$$

Fig. $2 \mathrm{j}$ shows a fit of this model to the $J(\mathrm{~V})$ data and reproduces all important observations: NDR, hysteretic switching, and the reset at positive bias (which 
involves the HOMO, Fig. 2k, n) (Supplementary Section S6). This modelling of the junction successfully describes the behaviour as shown by Figures $2 \mathrm{j}, 3 \mathrm{a}$ and $3 \mathrm{~g}$ and confirms that the appearance and disappearance of the mid-gap state causes switching and gives feedback on how and when the protonation occurs by giving an estimate for the switching probability (Fig.3g). To demonstrate that the presence of water is important, we repeated the experiment with relative humidity $<5 \%$ where no NDR effect is found (Fig.2i).

\section{Emulating synaptic behaviour}

To demonstrate basic synaptic function with our junctions, we perform pulse-pair facilitation (PPF) and depression (PPD) as follows. Fig.3a shows a typical pulse-pair sequence and current response. The difference in chordal conductance $\left(G=I_{\text {bias }} / V\right)$ between the measured and reference pulse in the pair, divided by the chordal conductance of the reference pulse, $\Delta G / G$, is used to quantify PPF and PPD, along with a modelled current response (more detail given in Supplementary Section S5.2). The magnitude of the voltage pulse $\left(V_{\mathrm{p}}\right)$, pulse duration $\left(t_{\mathrm{p}}\right)$, and time delay between consecutive pulses $\left(t_{\mathrm{d}}\right)$, programme the junction. For instance, Fig.3b shows that by changing $V_{\mathrm{p}}$ (with $\left.t_{\mathrm{p}}=t_{\mathrm{d}}=1 \mathrm{~s}\right)$ both conductance enhancement (excitatory potentiation) and depression (inhibitory potentiation) are obtained (Extended Data Fig. 2). Fig.3c shows that the extent of the potentiation depends on $t_{\mathrm{p}}$ where $\Delta G / G$ follows an exponential decay with characteristic time $2.4 \pm 0.9 \mathrm{~s}$ (Extended Data Fig. 3 and Supplementary Section S5.2.1.2).

Changing the delay between pulses provides different programming schemes. No significant decay is found in delays over minutes if the voltage is set at $0 \mathrm{~V}$ (Supplementary Section S5.2.1.3). However, the delay voltage can be set to a positive 
value, i.e., reset voltage $V_{\mathrm{r}}$, so that the junction will reset over time. Fig.3d shows that by changing $t_{\mathrm{d}}$ (with $V_{\mathrm{p}}=-2.0 \mathrm{~V}$ and $t_{\mathrm{p}}=1 \mathrm{~s}$ ) the conductance decreases exponentially with characteristic time of $\sim 2.5 \mathrm{~s}$ (Extended Data Fig. 4 and Supplementary Section S5.2.1.2). The speed of this reset process can be adjusted through the magnitude of $V_{\mathrm{r}}$ (Fig.3e and Extended Data Fig. 5). Fig.3d shows that spike timing dependent plasticity (STDP), where the order in which the pulses are applied and the delay between them modulates $\Delta G / G$, can also be emulated (Supplementary Section S5.2.1.2). The characteristic decay times of $12 \mathrm{~s}$ for positive $t_{\mathrm{p}}$ and $7 \mathrm{~s}$ for negative $t_{\mathrm{p}}$ are consistent with values obtained from our model (24s and 8 s respectively). Spike rate dependent plasticity (SRDP) is shown in Fig.3f. A negative inhibitory pulse $\left(V_{\mathrm{p}}=\right.$ $-1.0 \mathrm{~V})$ is applied at a set frequency while a positive excitatory voltage $\left(V_{\mathrm{d}}=1.25 \mathrm{~V}\right)$ is sustained during the delay between these pulses. Depending on the rate of the inhibitory pulse, the junction can have a net excitatory $(46 \mathrm{mHz})$ or inhibitory $(250$ $\mathrm{mHz}$ ) evolution over successive periods. These results demonstrate that, as in biological neural systems ${ }^{27,28}$, the frequency of the signal decides the (synaptic) junction response. This rate control provides a way to create discrete conductance states that can be recognised in subsequent cycles using several regular pulses. Fig.3g shows that junctions successfully reproduce discretisation of ten conductance states; this behaviour could be fully modelled. Repeated identical pulses do not potentiate the junction in a constant manner, instead the junctions show habituation, i.e., the junction desensitises to the signal as the response decays exponentially (as indicated in Fig.3f), mimicking how biological systems avoid overstimulation ${ }^{29}$. This habituation can be reversed through reset pulses and can be explained by the finite number of molecules electrically active in the junction, as captured by the model. 


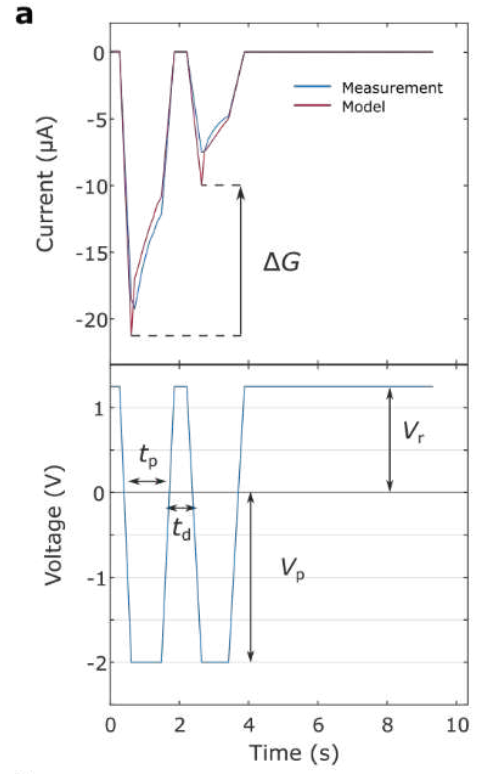

f

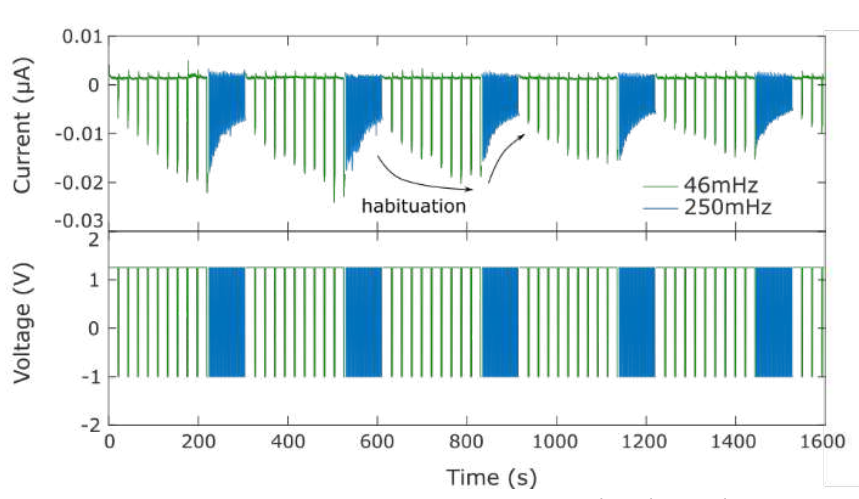

d
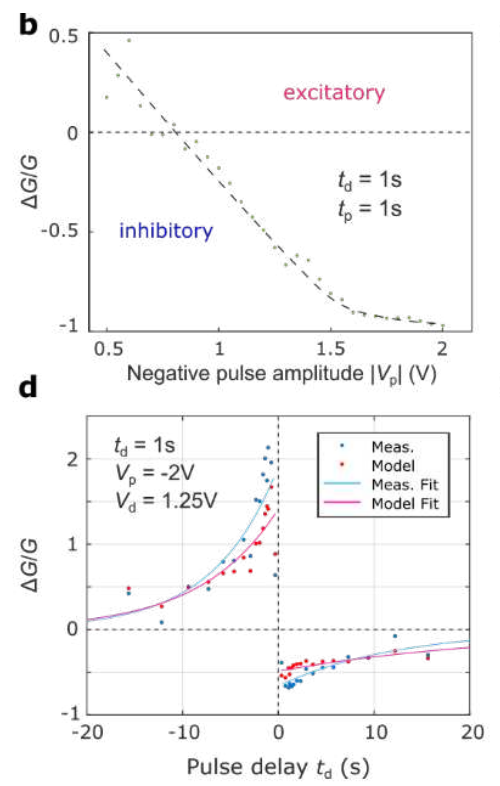

g

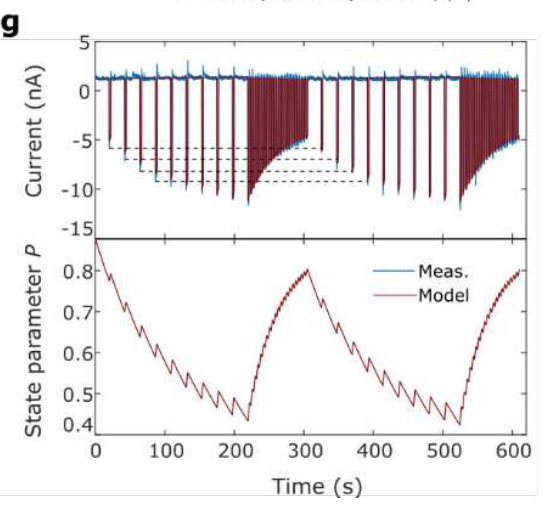

Fig. 3. Synaptic function. a, Typical voltage paired-pulse sequence and current response used to extract PPF and PPD properties, as measured (blue line) and modelled (red line) (additional fits are given in Supplementary Section S6.3). b-e, $\Delta G / G$ as a function of: (b) $-V_{\mathrm{p}},(\mathbf{c}) t_{\mathrm{p}}$, and (d) $t_{\mathrm{d}}$ with exponential fits (solid lines) used to extract characteristic time constants (Supplementary Sections S5.2.1.2 and S6.3), (e) $V_{\mathrm{r}}$, with dashed black lines as a visual guide. The pulse sequences and $I(V)$ profiles of the junctions used are shown in Extended Data Figures 2-5. f, Pulse sequence exhibiting SRDP with $V_{\mathrm{p}}=-1 \mathrm{~V}$ at $46 \mathrm{mHz}$ or $250 \mathrm{mHz}$ with the inter-pulse delay at $V_{\mathrm{d}}=1.25 \mathrm{~V}$. The solid black arrows indicate the effects of habituation. g, Identification and retrieval of conductance states over successive cycles and fit to the model. Dashed black lines are visual guides to show the recovery of the first four conductance states. The lower panel shows the evolution of dynamical switching probability $P$.

\section{Emulating Pavlovian learning and logic gates}

The junctions can be incorporated in electrical artificial neural networks (eANNs). Fig.4 shows an e-ANN that mimics Pavlov's experiment demonstrating the potential for the synapse to serve as a basis for Hebbian learning, i.e., training of an input by simultaneous operation with another input ('neurons that fire together, wire 
together ${ }^{14}$ ). Two inputs are fired from neurons N1 and N2 (here sources of voltage pulses) through synapses (electronic junctions) S1 and S2 respectively, to output neuron N3 (an ammeter), as shown in Fig.4a. In the Pavlov analogy, N1 represents 'dog sees food' which readily delivers an output in N3 'dog salivates' (Fig.4b). The sensitised synapse S1 between the two is materialised by a low-resistance resistor in the circuit. On the other hand, N2 (or 'dog hears bell') does not readily deliver the output in N3 (see insignificant output in Fig.4c, 'dog does not salivate'), but can be trained to do so through simultaneous operation of N1 and N2 (Fig.4d, 'dog sees food and hears bell'). This sensitises the adaptive synapse S2 (the dynamic switch) and subsequently enables N2 to produce an output in N3 (Fig.4e) (Extended Data Fig. 6). In this set-up, the whole Hebbian learning functionality is provided by the DMS, without the use of a third terminal and additional relays as reported before ${ }^{30}$

Components that can perform logic can be used as hidden layers in hardware deep-learning systems ${ }^{31}$. The time-domain plasticity of the DMS can be used to emulate all standard Boolean logic gates. While the DMS is a two-terminal component and standard logic gates require at least two inputs for one output, the time-domain plasticity of the DMS makes it possible to consider an applied voltage pulse as two separate inputs by separating the voltage and time domains in the pulse. The voltage magnitude of the pulse is one input and its duration a second input, and the resulting modulation in the conductance of the junction represents the output: positive if excitatory, and negative if inhibitory (Fig.4f-g). By defining the input states $V_{\text {on, }} V_{\text {off, }} t_{\text {on }}, t_{\text {off, }}$ the DMS can provide all the fundamental logic gates as shown in Fig.4h-I and Extended Data Fig. 7 (Supplementary Section S5.2.3). 

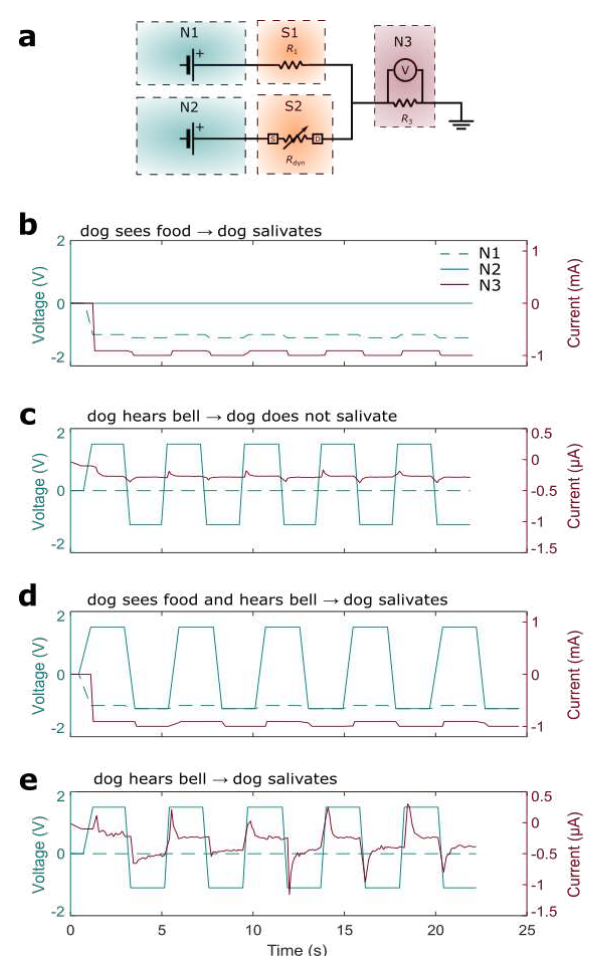
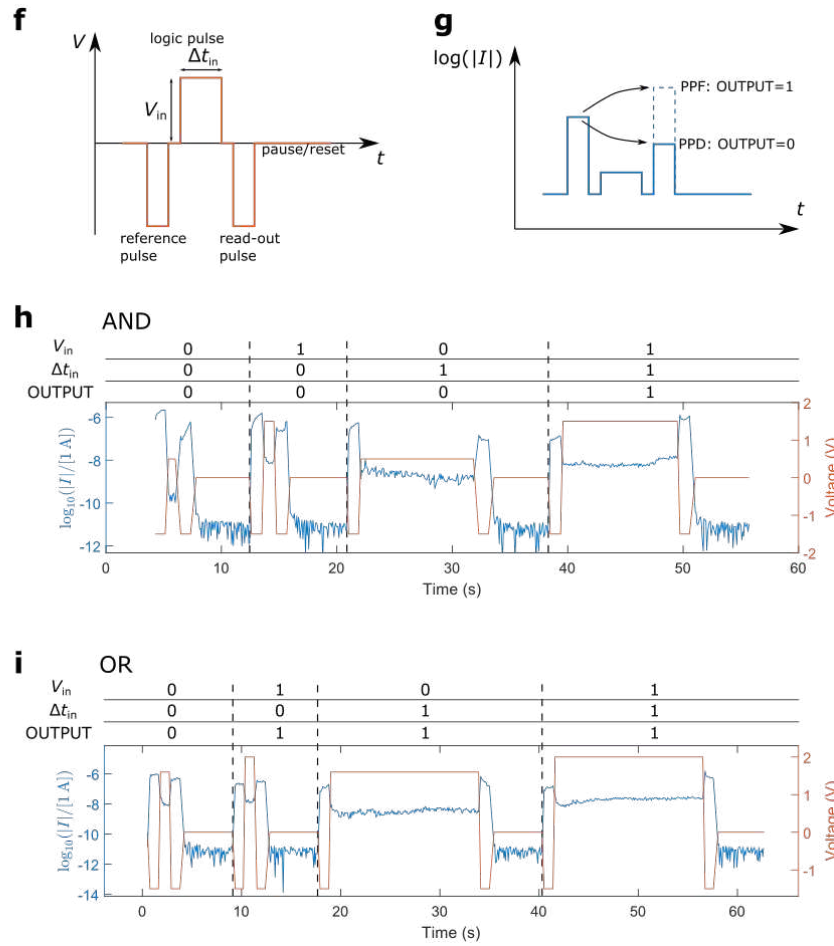

Fig.4. Demonstration of Pavlovian learning and Boolean logic gates using time plasticity in the DMS. a, Schematic of the electrical circuit. Two input neurons N1 and N2 are connected to an output neuron N3 via synapses S1 (resistor) enabling strong coupling between N1 and N3, and S2 (DMS), the trainable synapse. b-e, Pulse sequences showing Pavlovian learning. b, Pulse sequence representing 'dog sees food', pulses from N1 produce a strong output in N3. c, 'dog hears bell' before training, N2 produces an insignificant output in N3. d, 'dog sees food and hears bell', which trains S2. Pulses from N1 and N2 combined produce a strong output in N3. e, 'dog hears bell' after training, N2 now produces a significant output in N3. f, A reference voltage pulse is followed by the logic pulse to perform the operation and finally a read-out pulse. g, The current read-out pulse is compared to the reference pulse, where current increase is defined as output $=1$ and current decrease as output $=0$. Demonstration of the AND (h) and OR (i) logic gates. All other gates and input parameters are shown in Extended Data Fig. 7 and Supplementary Section S5.2.3.

\section{Conclusions}

We report a dynamic molecular switch (DMS) of just $2.4 \mathrm{~nm}$ that readily emulates the plasticity of synapses, exhibits Pavlovian learning, and can be configured to perform all logic gates required for deep learning, all within one junction. This multifunctionality combined with reconfigurability has the potential to simply braininspired electronic devices considerably. The switching probability of the junction changes dynamically between high and low conduction states enabled via PC-ET 
driven dynamic $\mathrm{N}-\mathrm{H}$ bond formation. A challenge is usually to understand the microscopic origin of the dynamics of mesoscale artificial synapses, and such systems are usually modelled with equivalent circuit analysis or network models. In contrast, the dynamical behaviour of our DMS can be readily captured with intertwined rate equations with different time constants and can be applied to dynamic molecular systems driven by other stimuli ( dynamic covalent bond formation ${ }^{33}$ opening up a whole new range of adaptive and reconfigurable systems with orthogonal inputs and outputs.

\section{References}

1. Van De Burgt, Y., Melianas, A., Keene, S. T., Malliaras, G. \& Salleo, A. Organic electronics for neuromorphic computing. Nat. Electron. 1, 386-397 (2018).

2. Zhu, J., Zhang, T., Yang, Y. \& Huang, R. A comprehensive review on emerging artificial neuromorphic devices. Appl. Phys. Rev. 7, 011312 (2020).

3. Upadhyay, N. K. et al. Emerging Memory Devices for Neuromorphic Computing. Adv. Mater. Technol. 4, 1800589 (2019).

4. Chen, T. et al. Classification with a disordered dopant-atom network in silicon. Nature 577, 341-345 (2020).

5. Keene, S. T. et al. A biohybrid synapse with neurotransmitter-mediated plasticity. Nat. Mater. 19, 969-973 (2020).

6. Oh, S., Hwang, H. \& Yoo, I. K. Ferroelectric materials for neuromorphic computing. APL Mater. 7, 91109 (2019).

7. Wang, L., Lu, S. R. \& Wen, J. Recent Advances on Neuromorphic Systems Using Phase-Change Materials. Nanoscale Research Letters 12, 1-22 (2017). 
8. Wan, Q., Sharbati, M. T., Erickson, J. R., Du, Y. \& Xiong, F. Emerging Artificial Synaptic Devices for Neuromorphic Computing. Adv. Mater. Technol. 4, 1900037 (2019).

9. Ratera, I. \& Veciana, J. Playing with organic radicals as building blocks for functional molecular materials. Chem. Soc. Rev. 41, 303-349 (2012).

10. Klajn, R. Spiropyran-based dynamic materials. Chem. Soc. Rev. 43, 148-184 (2014).

11. Bléger, D. \& Hecht, S. Visible-Light-Activated Molecular Switches. Angew. Chemie - Int. Ed. 54, 11338-11349 (2015).

12. Sorrenti, A., Leira-Iglesias, J., Markvoort, A. J., De Greef, T. F. A. \& Hermans, T. M. Non-equilibrium supramolecular polymerization. Chem. Soc. Rev. 46, 5476-5490 (2017).

13. Van Rossum, S. A. P., Tena-Solsona, M., Van Esch, J. H., Eelkema, R. \& Boekhoven, J. Dissipative out-of-equilibrium assembly of man-made supramolecular materials. Chem. Soc. Rev. 46, 5519-5535 (2017).

14. Bear, M. F., Connors, B. W. \& Paradiso, M. A. Neuroscience: Exploring the Brain. (Wolters Kluwer, 2016).

15. Eccles, J. C. \& McIntyre, A. K. Plasticity of mammalian monosynaptic reflexes. Nature 167, 466-468 (1951).

16. Segura, J. L., Juárez, R., Ramos, M. \& Seoane, C. Hexaazatriphenylene (HAT) derivatives: From synthesis to molecular design, self-organization and device applications. Chem. Soc. Rev. 44, 6850-6885 (2015).

17. Wang, R. et al. Cyclic and normal pulse voltammetric studies of 2,3,6,7,10,11hexaphenylhexazatriphenylene using a benzonitrile thin layer-coated glassy carbon electrode. J. Phys. Chem. B 107, 9452-9458 (2003). 
18. Egger, D. A., Liu, Z. F., Neaton, J. B. \& Kronik, L. Reliable Energy Level Alignment at Physisorbed Molecule-Metal Interfaces from Density Functional Theory. Nano Lett. 15, 2448-2455 (2015).

19. Perrin, M. L. et al. Large negative differential conductance in single-molecule break junctions. Nat. Nanotechnol. 9, 830-834 (2014).

20. Migliore, A. \& Nitzan, A. Irreversibility and hysteresis in redox molecular conduction junctions. J. Am. Chem. Soc. 135, 9420-9432 (2013).

21. Schwarz, F. et al. Field-induced conductance switching by charge-state alternation in organometallic single-molecule junctions. Nat. Nanotechnol. 11, 170-176 (2016).

22. Garrigues, A. R. et al. A Single-Level Tunnel Model to Account for Electrical Transport through Single Molecule-and Self-Assembled Monolayer-based Junctions. Sci. Rep. 6, 26517 (2016).

23. Fung, E. D. et al. Breaking Down Resonance: Nonlinear Transport and the Breakdown of Coherent Tunneling Models in Single Molecule Junctions. Nano Lett. 19, 2555-2561 (2019).

24. Warren, J. J. \& Mayer, J. M. Chapter 1 Application of the Marcus Cross Relation to Hydrogen Atom Transfer/Proton-Coupled Electron Transfer Reactions. in Proton-Coupled Electron Transfer: A Carrefour of Chemical Reactivity Traditions 1-31 (The Royal Society of Chemistry, 2012). doi:10.1039/9781849733168-00001

25. Migliore, A., Polizzi, N. F., Therien, M. J. \& Beratan, D. N. Biochemistry and theory of proton-coupled electron transfer. Chem. Rev. 114, 3381-3465 (2014).

26. Mayer, J. M. Understanding hydrogen atom transfer: From bond strengths to marcus theory. Acc. Chem. Res. 44, 36-46 (2011). 
27. Kim, S. et al. Experimental demonstration of a second-order memristor and its ability to biorealistically implement synaptic plasticity. Nano Lett. 15, 2203$2211(2015)$.

28. Wang, Z. et al. Memristors with diffusive dynamics as synaptic emulators for neuromorphic computing. Nat. Mater. 16, 101-108 (2017).

29. Thompson, R. Habituation. in International Encyclopedia of the Social \& Behavioral Sciences 6458-6462 (Pergamon, 2001). doi:10.1016/B0-08043076-7/03639-1

30. Van De Burgt, Y. et al. A non-volatile organic electrochemical device as a lowvoltage artificial synapse for neuromorphic computing. Nat. Mater. 16, 414418 (2017).

31. Ruiz Euler, H. C. et al. A deep-learning approach to realizing functionality in nanoelectronic devices. Nat. Nanotechnol. 15, 992-998 (2020).

32. Kathan, M. et al. Light-driven molecular trap enables bidirectional manipulation of dynamic covalent systems. Nat. Chem. 10, 1031-1036 (2018).

33. Chakma, P. \& Konkolewicz, D. Dynamic Covalent Bonds in Polymeric Materials. Angew. Chemie - Int. Ed. 58, 9682-9695 (2019). 


\section{Methods}

Synthesis. 5,6,11,12,17,18-hexaazatrinaphthylene (HATNA) was synthesised following the similar procedure reported previously ${ }^{34}$, the detailed synthesis scheme and characterisation of molecule are summarised in Supplementary Section S2.

Electrode preparation. The cone-shaped $\mathrm{GaOx} / \mathrm{EGaIn}$ top-electrode was formed using previously reported methods ${ }^{35}$. The gold bottom-electrode was prepared following reported template stripping methods ${ }^{36}$. We deposited $200 \mathrm{~nm}$ thick Au films (containing 99.999\% Au granules, ACI Alloys) on commercial Si (100) wafers (with a native $\mathrm{SiO}_{2}$ thin layer on surface) using a Shen Yang Ke Yi thermal evaporator. Clean glass slides were then glued onto the Au surface with thermal glue (EPOTEK 353ND) and cured at $80{ }^{\circ} \mathrm{C}$ for 3 hours. To minimise contamination from the atmosphere, the wafers coated with $\mathrm{Au}$ were stored in a dry box and the Au was template-stripped immediately before use.

Self-assembled monolayer (SAM) formation on $\mathbf{A u}^{\mathrm{TS}}$. The S-C10-HATNA SAMs were formed using well-established methods ${ }^{35}$. Typically, $1.0 \mathrm{mg}$ HS-C $10-H A T N A$ was dissolved in freshly distilled THF $(6 \mathrm{ml})$ to obtain a solution with a concentration of $0.2-0.3 \mathrm{mM}$. The solution was degassed for $15 \mathrm{~min}$ with $\mathrm{N}_{2}$ to remove oxygen, and then a freshly template-stripped Au surface was immersed into the solution for $24 \mathrm{~h}$ under an atmosphere of $\mathrm{N}_{2}$. The coated Au substrates were then removed from the solution and washed with THF and ethanol to remove physisorbed molecules and gently dried in a flow of $\mathrm{N}_{2}$. 
Electrochemistry. The S-C $10-\mathrm{HATNA}$ SAMs on $\mathrm{Au}$ were electrochemically characterised by cyclic voltammetry. We used a custom-built electrochemical cell equipped with a $\mathrm{Pt}$ counter electrode, $\mathrm{Ag} / \mathrm{AgCl}$ reference electrode and $\mathrm{Au}^{\mathrm{TS}}$ working electrode. $\mathrm{A} \mathrm{HClO}_{4}$ aqueous solution $(2 \mathrm{M})$ was used as the electrolyte, similar to a previous report ${ }^{17}$. The cyclic voltammograms were recorded between $-0.3 \mathrm{~V}$ and 0.8 $\mathrm{V}$ at scan rates ranging from $0.05 \mathrm{~V} / \mathrm{s}$ to $10 \mathrm{~V} / \mathrm{s}$ with an Autolab PGSTAT302T equipped with NOVA 1.10 software (Supplementary Section S3).

Surface characterisation. Synchrotron-based photoemission spectroscopy, including X-ray photoelectron spectroscopy (XPS), ultraviolet photoelectron spectroscopy (UPS) and near-edge X-ray absorption fine structure (NEXAFS) spectroscopy were performed using the Surface, Interface and Nanostructure Science (SINS) beamline of the Singapore Synchrotron Light Source (SSLS). The experimental procedures are described in our previous work ${ }^{37}$. We measured the SAMs of S-C10-HATNA on template-stripped Au surfaces. For high-resolution XPS, we used photon energies of $350 \mathrm{eV}$ for $\mathrm{S} 2 p$ and $\mathrm{C} 1 s, 600 \mathrm{eV}$ for $\mathrm{N} 1 s$ and $650 \mathrm{eV}$ for $\mathrm{O} 1 s$ spectra for optimal photoionisation cross-sections at these core-levels. We recorded each XPS signal with two different photoelectron take-off angles, $90^{\circ}$ (normal emission, NE) and $40^{\circ}$ (grazing emission, GE). We performed peak fitting using pseudo-Voigt functions with a fixed $70 \%$ Gaussian and $30 \%$ Lorentzian ratio and a Shirley background. The detailed results of surface characterisation were shown in Supplementary Section S3.

DFT electronic structure calculations. We calculated the density of states (DOS) distributions for the HS-C10-HATNA and the reduced state HS-C10-(H6-HATNA) and all intermediate states along the PC-ET path using previously reported methods ${ }^{38}$. All 
computed reorganisation energies of HATNA during sequential PC-ET steps, the calculated PDOS of Au-S-C $10-\mathrm{HATNA}$ and post-PCET Au-S-C10-(H6-HATNA), the computed surfaces of the electron acceptor mid-gap and LUMO $\rightarrow \mathrm{LUMO}+2$ levels, and the calculated PDOS on the molecule of HATNA SAMs on Au(111) for all intermediate oxidation and protonated states are summarised in Supplementary Section S4.

Junction fabrication and electrical measurements in air. We formed electrical contacts to the SAMs with cone-shaped tips of GaOx/EGaIn using previously reported methods ${ }^{35}$. All measurements were performed with the bottom contact connected to the ground and the bias was applied to the EGaIn top electrode from $0 \mathrm{~V}$ $\rightarrow+1 \mathrm{~V} \rightarrow 0 \mathrm{~V} \rightarrow-2 \mathrm{~V} \rightarrow 0 \mathrm{~V}$. A Keithley 6430 source meter was used to bias the junction controlled by LabVIEW. The $J(\mathrm{~V})$ curves were recorded in steps of $20 \mathrm{mV}$ (Supplementary Section S5).

Statistical analysis of $\boldsymbol{J}(\mathrm{V})$ characteristics. All $J(\mathrm{~V})$ data were used to perform the statistical analysis excepting the unstable and shorted junctions. We define a junction as unstable when the current value suddenly increased to the compliance limit of our electrometer. The number of consecutive $J(\mathrm{~V})$ traces is limited to 3 traces per junction because of the memory effect on the switching probabilities of the molecules (as explained in the main text). We determined the median averages of the values of $\log _{10}|J|$ along with the median absolute deviations, $\sigma_{\mathrm{m}}$, a recommended alternative method as described in ref $^{39}$, because this method does not rely on assumptions regarding the distribution of the data. From all $\log _{10}|J|$ values measured at each bias, we calculated the median $\log _{10}|J|$ average, $\left\langle\log _{10}|J|>_{\mathrm{m}}\right.$, and $\sigma_{\mathrm{m}}$. We created our 
heatmaps using 2D kernel density estimations with OriginPro 2019b. We used exact estimation as the density calculation method, bivariate kernel density estimator as bandwidth selection method and a grid size of 100. Exact estimation method in OriginPro $2019 \mathrm{~b}$ calculates the density values based on a bidimensional Gaussian kernel. See the detailed description in Supplementary Section S5.

Synaptic behaviour, Pavlovian learning, and logic gates. The measurements of the junctions as an artificial synapse were carried out with samples prepared using methods as described in the SAM formation section in the Methods and using a coneshaped tip of $\mathrm{GaOx} / \mathrm{EGaIn}$ as described in the Electrodes preparation section in the Methods. For each junction an initial $I(V)$ measurement was performed as described in the Junction fabrication and electrical measurements section in the Methods to ensure the junction had $J(V)$ characteristics close to the log-median (Fig. S7), as given in Supplementary Information Section S5.2. Following this, sequences of voltage pulses were programmed and applied using LabView-based software CryoMeas (developed by Prof C.J.B. Ford, University of Cambridge) using a Keithley 6430 source meter which recorded the current simultaneously. A typical pulse sequence used to extract plasticity depending on pulse parameters is shown in Fig. 3a, while the custom pulse sequences used for Pavlovian learning are shown and described in Supplementary Information Section S5.2. Pavlovian learning was demonstrated in a set-up involving two Keithley 6430 source meters operated simultaneously as described in the main text (Fig. 4a-e) and Extended Data Fig. 6 Supplementary Information Section S5.2.2. Pulse parameters used for the logic gates are described in Supplementary Information Section S5.2.3. 
Theoretical Modelling of the Transport Dynamics. We have used a theoretical model developed by Migliore and Nitzan ${ }^{20}$ to model the junction characteristics and explain our observations. We used the standard single-level quantum model to account for the current through the molecular junction in both the on and off states which has been shown to effectively describe the conduction through single-molecule junctions, which has been modified by us to describe the current across large-area molecular junctions ${ }^{35,40}$. The model described above has been used to fit the data presented in Figs. 3d-h in the main text. The detailed description of the mechanistic modelling, Landauer formalism to account for the tunnelling in the on and off states, fitting of the data and the related discussion are given in Supplementary Section S6.

Data availability: Data and codes are available in the supplementary materials and on https://dataverse.harvard.edu/privateurl.xhtml?token=efd1a016-cc24-41ab-891b$5217 \mathrm{fa} 6 \mathrm{dd} 56 \mathrm{~d}$.

\section{References}

34. Larsen, C. B. et al. Synthesis and Optical Properties of Unsymmetrically Substituted Triarylamine Hexaazatrinaphthalenes. European J. Org. Chem. 2017, 2432-2440 (2017).

35. Chen, X. et al. Molecular diodes with rectification ratios exceeding $10^{\wedge} 5$ driven by electrostatic interactions. Nat. Nanotechnol. 12, 797-803 (2017).

36. Yuan, L., Jiang, L., Thompson, D. \& Nijhuis, C. A. On the remarkable role of surface topography of the bottom electrodes in blocking leakage currents in molecular diodes. J. Am. Chem. Soc. 136, 6554-6557 (2014).

37. Yuan, L., Breuer, R., Jiang, L., Schmittel, M. \& Nijhuis, C. A. A Molecular 
Diode with a Statistically Robust Rectification Ratio of Three Orders of Magnitude. Nano Lett. 15, 5506-5512 (2015).

38. Han, Y. et al. Electric-field-driven dual-functional molecular switches in tunnel junctions. Nat. Mater. 19, 843-848 (2020).

39. Reus, W. F. et al. Statistical tools for analyzing measurements of charge transport. J. Phys. Chem. C 116, 6714-6733 (2012).

40. Yuan, L. et al. Transition from direct to inverted charge transport Marcus regions in molecular junctions via molecular orbital gating. Nat. Nanotechnol. 13, (2018).

\section{ACKNOWLEDGEMENTS}

We thank the Ministry of Education (MOE, Award No. MOE2018-T2-1-088 and MOE2019-T2-1-137) and the Prime Minister's Office, Singapore, under its Medium Sized Centre program for supporting this research. D.T. acknowledges support from Science Foundation Ireland (SFI) under awards number 15/CDA/3491 and 12/RC/2275_P2 and supercomputing resources at the SFI/Higher Education Authority Irish Center for High-End Computing (ICHEC). E.d.B. and C.N. acknowledge support from the U.S. National Science Foundation (Grant no. ECCS\#1916874). D.Q. acknowledges the support of the Australian Research Council (Grant No. FT160100207).

\section{Author contributions}

C.A.N. conceived and supervised the project. D.T. conducted the atomistic modelling. E.d.B. and C.N. conducted the numerical modelling. Y.W. synthesised the compounds, Y.W. and Y.H. performed the CV and UV-vis measurements. Z.Z. and 
D.Q. performed the AR-XPS and NEXAFS measurements and analysis. Y.W. and Q.Z. performed the $I-V$ electrical measurements. H.P.A.G.A. designed, performed and analysed the synaptic emulation measurements. A.L. developed the Origin code for electrical data analysis. C.A.N., E.d.B., D.T., and H.P.A.G.A. wrote the manuscript and all authors commented on it.

Competing interests: The authors declare no competing interests.

\section{Additional information}

Extended data is available for this paper at https://doi.org/xxx.

Supplementary information is available for this paper at https://doi.org/xxx.

Correspondence and requests for materials should be addressed to E.d.B., D.T. or C.A.N.

Reprints and permissions information is available at www.nature.com/reprints. 


\section{Extended Data}
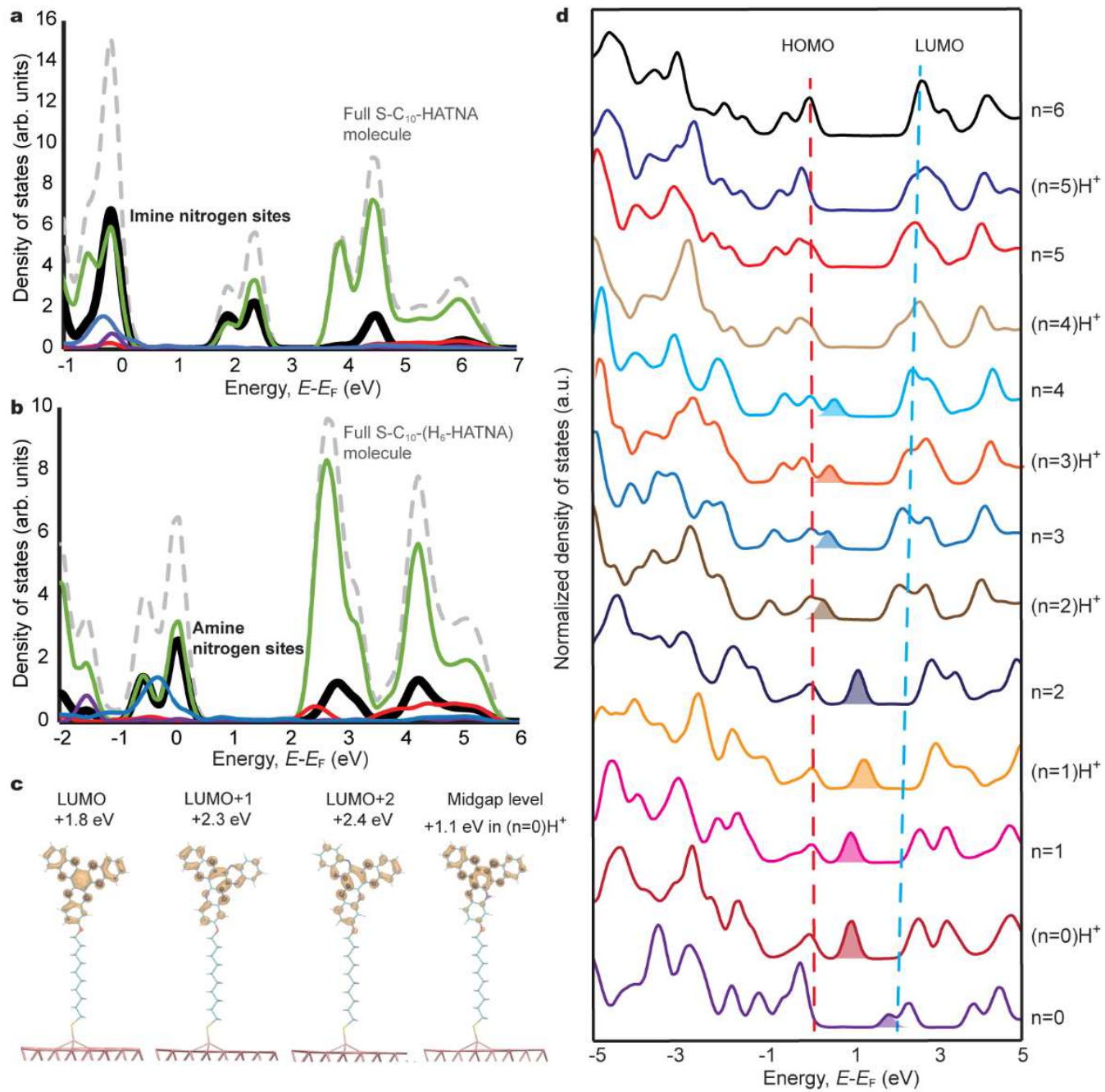

Extended Data Fig. 1. The periodic DFT calculations. a, The calculated PDOS of Au-bound S-C 10 -HATNA with the nitrogen sites plotted as a thick black line. The full molecule is shown as a dashed grey line and contributions from carbon, hydrogen, oxygen, and sulphur sites are plotted as green, red, purple, and blue lines, respectively. b, The calculated PDOS of post-PCET Au-S-C $\mathrm{C}_{10}-\left(\mathrm{H}_{6}-\mathrm{HATNA}\right)$. (c) Computed surfaces of the empty electron-acceptor LUMO $\rightarrow$ LUMO +2 levels in AuS-C 10 -HATNA together with the mid-gap state created at $+1.1 \mathrm{eV}$ in the first protonation step generating Au-S-C $10-\mathrm{H}^{+}$-HATNA. d, The calculated PDOS on the molecule of HATNA SAMs on $\mathrm{Au}(111)$ for all oxidation and protonated states. 

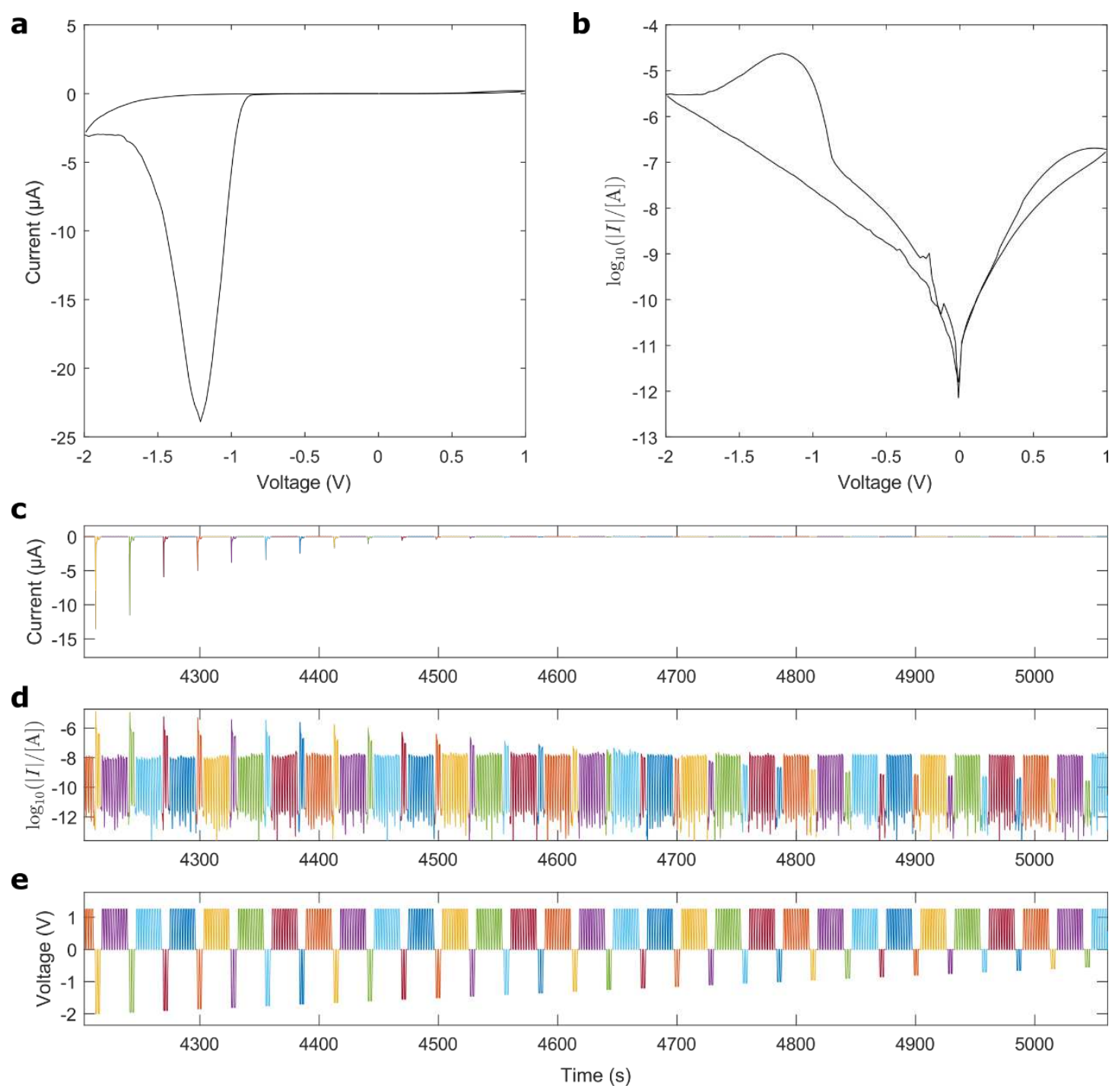

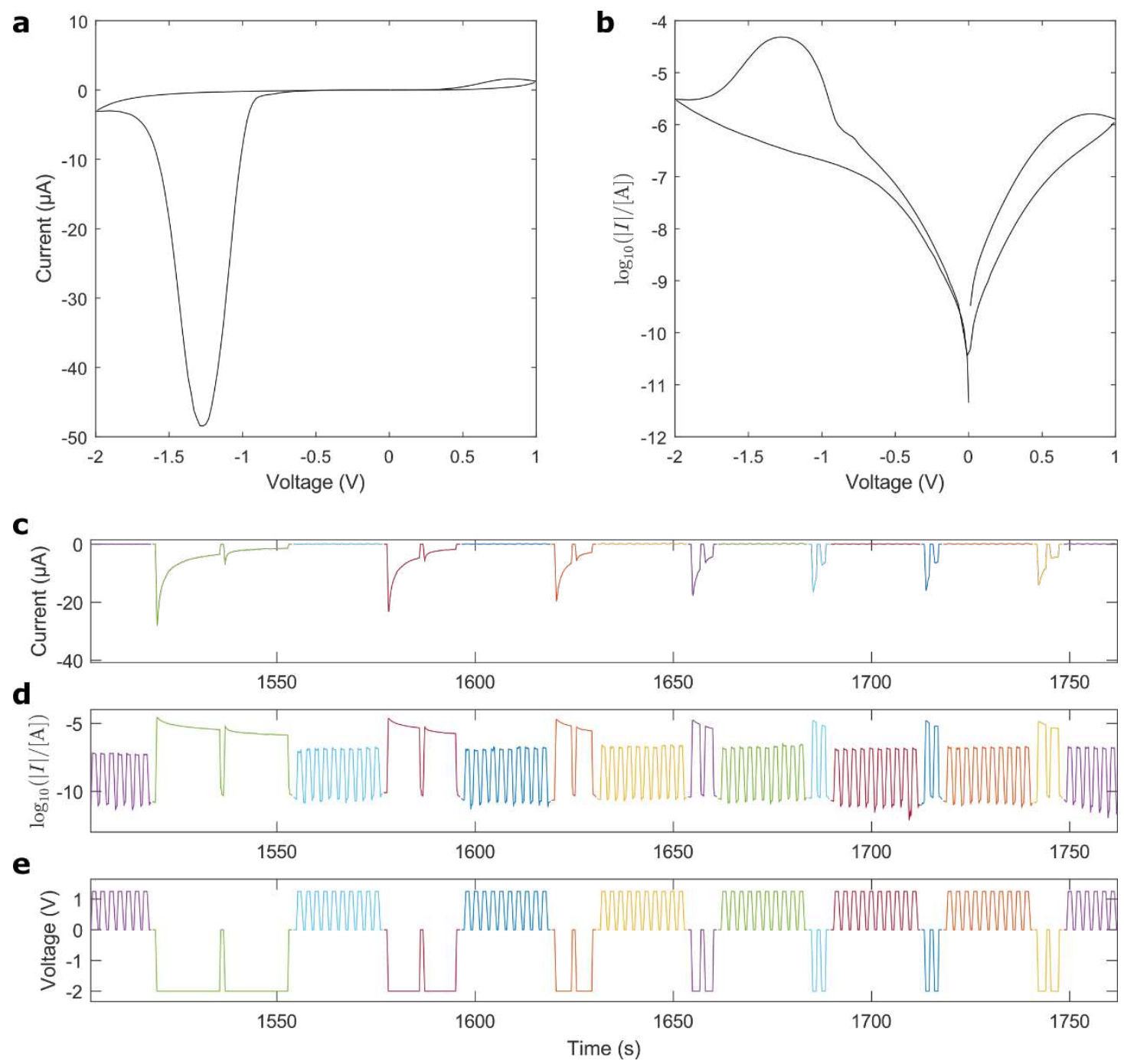

Extended Data Fig. 3. Measurement of potentiation as a function of negative pulse duration as shown in Fig. 3c. a-b, $I(V)$ profile of the junction used in the measurement in linear (a) and logarithmic scale (b). c-e, pulse sequence used in the measurement with current (c), logarithm of current (d) and voltage (e) as a function of time, with colour used to help distinguish sections of the sequence. A first negative voltage pulse of amplitude $V_{\mathrm{p}}=-2 \mathrm{~V}$ and duration $t_{\mathrm{p}}$ was applied, a delay at $0 \mathrm{~V}$ was set for $t_{\mathrm{d}}=1 \mathrm{~s}$, a second negative voltage pulse of magnitude $V_{\mathrm{p}}$ was applied, followed by a series of positive voltage pulses of amplitude $V_{\mathrm{r}}=1.25 \mathrm{~V}$ to reset the junction. This sequence was repeated each time varying $V_{\mathrm{p}}$ the amplitude of the negative voltage pulses from $0.5 \mathrm{~V}$ to $2 \mathrm{~V}$ and back, and after half a sequence to train the junction, the current amplitude of the second negative pulse was compared to that of the first negative pulse to determine the potentiation as a function of $V_{\mathrm{p}}$. 

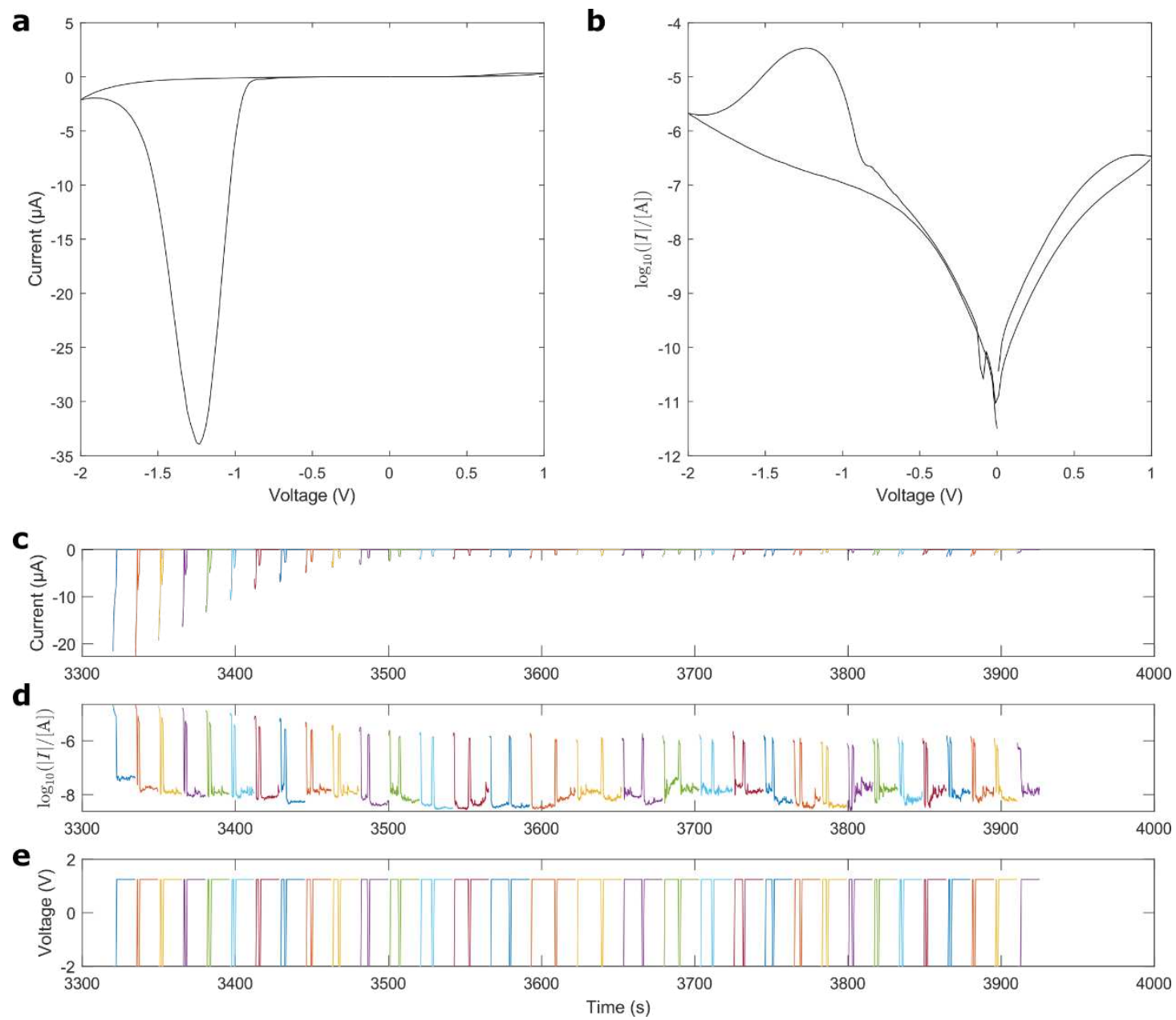

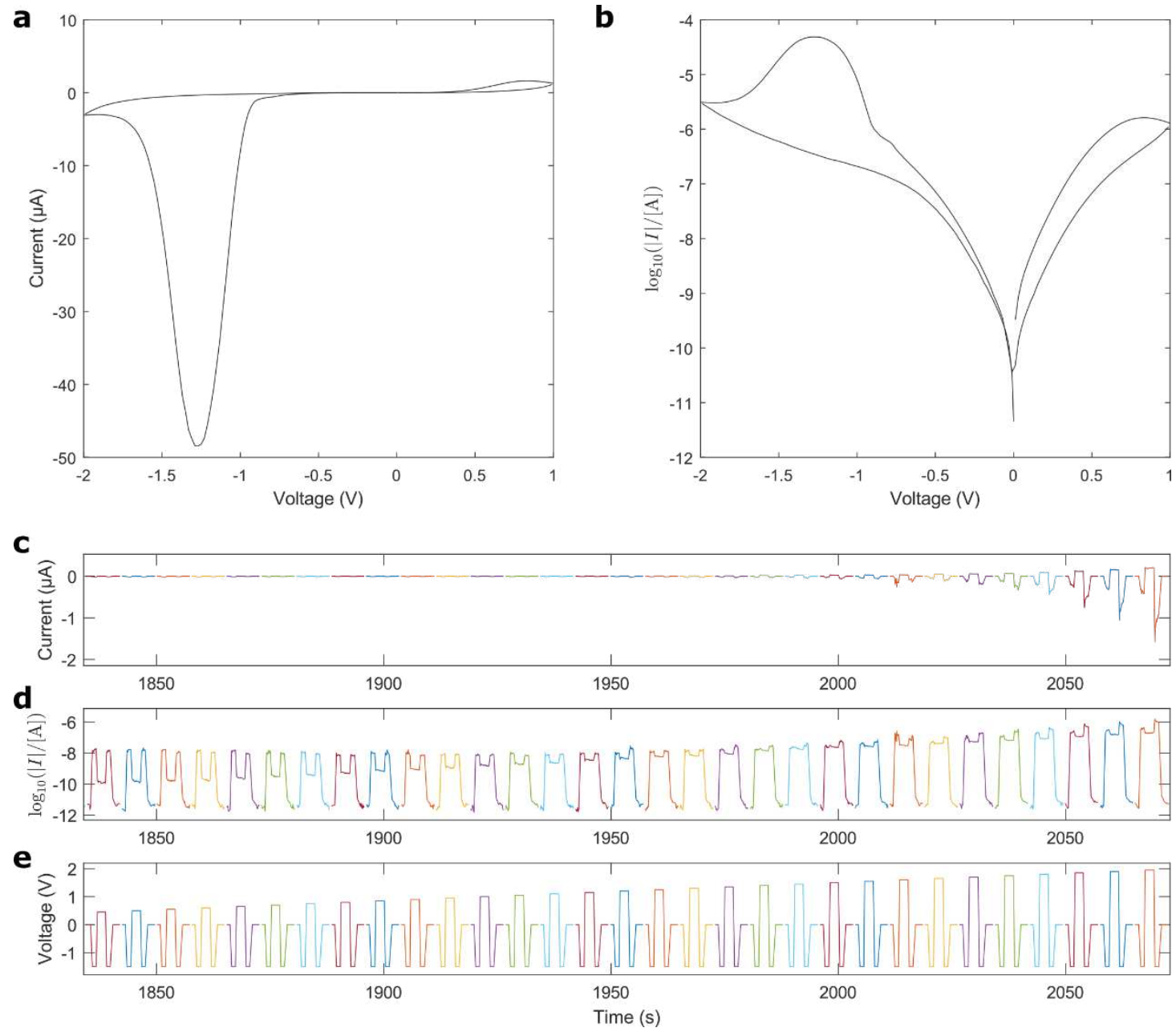

Extended Data Fig. 5. Measurement of potentiation as a function of positive pulse duration as shown in Fig. 3e. a-b, $I(V)$ profile of the junction used in the measurement in linear (a) and logarithmic scale (b). c-e, pulse sequence used in the measurement with current (c), logarithm of current (d) and voltage (e) as a function of time, where colour is used to help distinguish sections of the sequence. A negative reference voltage pulse $\left(V_{\mathrm{p}}=-1.5 \mathrm{~V}, t_{\mathrm{p}}=1 \mathrm{~s}\right)$ was applied, a positive voltage pulse of varying amplitude (duration $t_{\mathrm{d}}=2 \mathrm{~s}$ ) was applied, and a second negative voltage pulse $\left(V_{\mathrm{p}}=-\right.$ $\left.1.5 \mathrm{~V}, t_{\mathrm{p}}=1 \mathrm{~s}\right)$ was applied. This sequence was repeated each time varying $V_{\mathrm{r}}$ the amplitude of the positive voltage pulse from $0.5 \mathrm{~V}$ to $2 \mathrm{~V}$ and back, and after one training sequence $(\sim 500 \mathrm{~s})$, the current amplitude of the second negative pulse was compared to that of the reference negative pulse to determine the potentiation as a function of $V_{\mathrm{r}}$. 

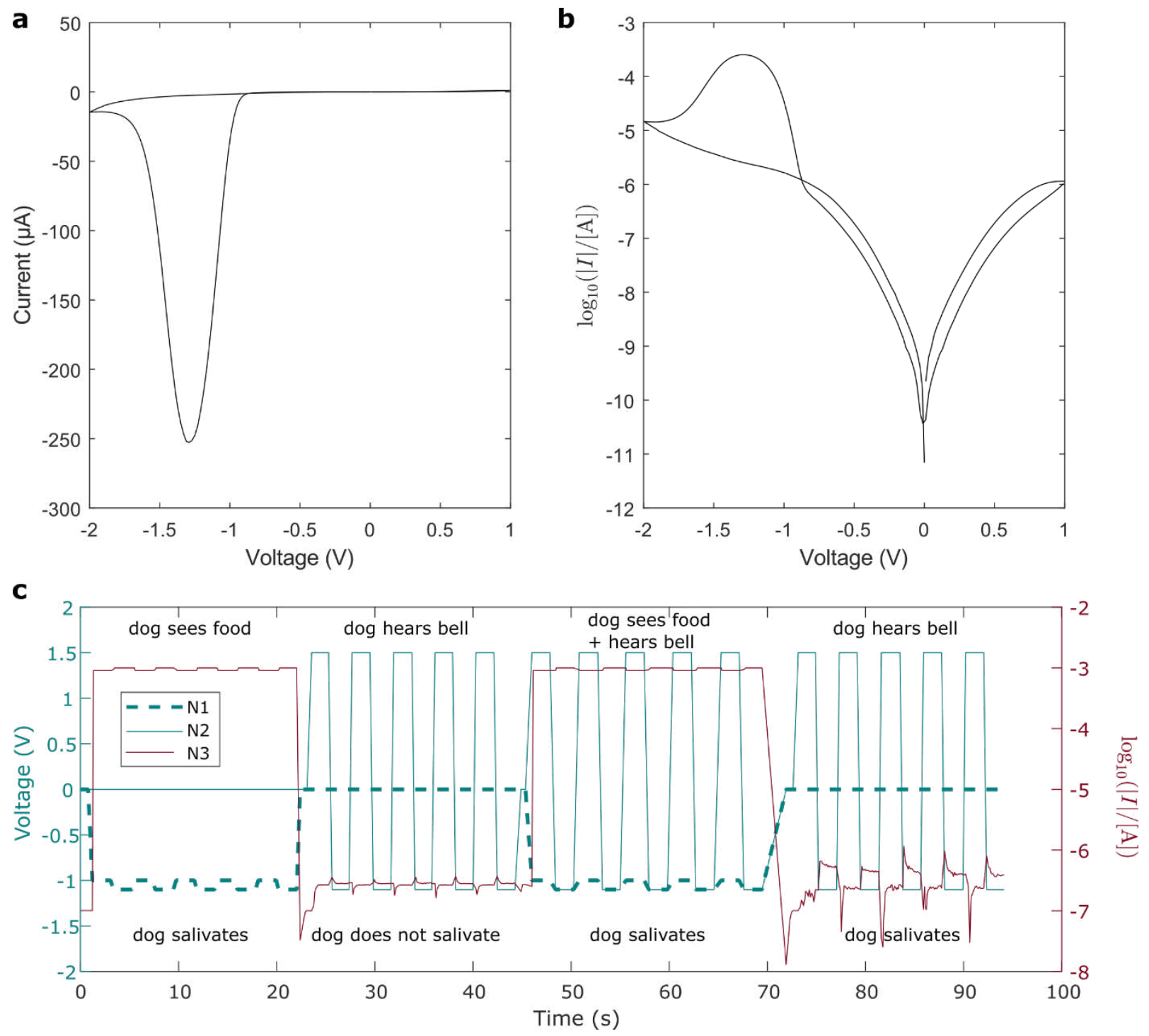

Extended Data Fig. 6. Measurement demonstrating the Pavlov experiment (Hebbian learning). a-b, $I(V)$ profile of the junction used in the measurement in linear (a) and logarithmic scale (b). c, pulse sequence showing Hebbian learning (from Fig. 4b-e). The e-ANN works as follows: two source-measure units represent two artificial neurons $\mathrm{N} 1$ and $\mathrm{N} 2$ delivering voltage inputs in separate channels feeding into a third channel (see Fig. 4a) N3 where the current received is the output. N1 is connected via a fixed resistor $(1 \mathrm{k} \Omega)$ to $\mathrm{N} 3$ and gives the output unconditionally. N2 includes the junction in the off state such that pulses from N2 do not initially result in a significant signal in N3. It corresponds to 'dog hears bell'. However, firing pulses in N1 and N2 results in a PPF process in N2, 'training' it to deliver an output in N3 when pulses are fired from $\mathrm{N} 2$ thereafter. The sequences of voltage pulses are as follows. N1 alternates between pulses at $-1 \mathrm{~V}$ and $-1.1 \mathrm{~V}$, each lasting $2 \mathrm{~s}$, repeated 5 times. $\mathrm{N} 2$ alternates between pulses at $+1.5 \mathrm{~V}$ and $-1.1 \mathrm{~V}$, each lasting $2 \mathrm{~s}$, repeated 5 times. When pulses are applied from N1 and N2 simultaneously, the voltage across the dynamic switch can be estimated to be alternating between $+2.4 \mathrm{~V}$ and $-0.1 \mathrm{~V}$ approximately, according to Kirchhoff's circuit laws, which excites the junction (enhances its conductance) and thus the coupling to N3. 

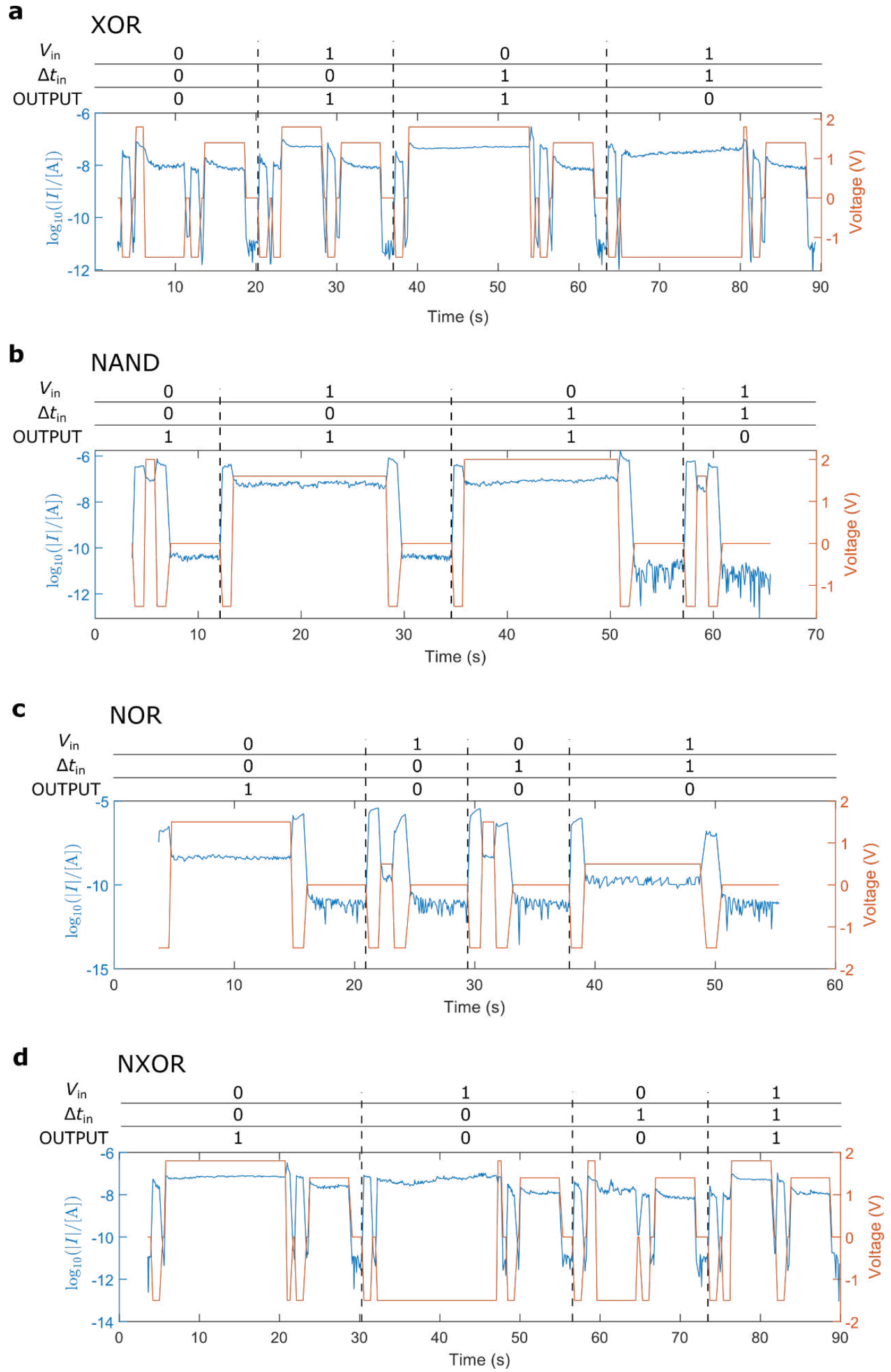

Extended Data Fig. 7. Demonstration of remaining logic gates: a, XOR; b, NAND; c, NOR; d, NXOR, as per the operation system described in the main text and Fig. 4, with current and voltage traces and truth tables. More details on the pulse parameters are given in Supplementary Information Section S5.2.3. 


\section{Supplementary Files}

This is a list of supplementary files associated with this preprint. Click to download.

- supportinginformationfile.docx 